\title{
Human papillomavirus genotype distribution in cervical samples collected in routine clinical practice at the Nantes University Hospital, France
}

\author{
Edouard Vaucel • Marianne Coste-Burel • \\ Christian Laboisse $\cdot$ André Dahlab $\cdot$ Patrice Lopes
}

Received: 24 April 2010 / Accepted: 21 October 2010 / Published online: 25 November 2010

(C) The Author(s) 2010. This article is published with open access at Springerlink.com

\begin{abstract}
Objective The objective was to assess the human papillomavirus (HPV) overall and type-specific prevalence in smears collected during routine clinical practice.

Design HPV genotyping and smears were performed independently between 2000 and 2006 for routine clinical follow-up (primary screening and follow-up) in the University Hospital of Nantes, France.

Population All women with a cytological sample collected no more than 12 months before HPV genotyping were included.

Methods PCR was performed with MY09/MY11 primers and genotyping by sequencing PCR product.

Main outcome measures Overall and genotype-specific HPV prevalence were assessed according to cytological diagnosis.

Results A total of 1,255 women were included (mean age 37.5 years). The proportion of high-risk (HR) HPV positive samples increased according to cytological diagnosis severity from $8 \%$ in normal specimens to $21 \%$ in atypical
\end{abstract}

E. Vaucel · P. Lopes $(\bowtie)$

CHU de Nantes, Pôle Mère-Enfant,

Service de Gynécologie Obstétrique et Médecine de la

Reproduction, 7 quai Moncousu, 44093 Nantes Cedex 1, France

e-mail: patrice.lopes@chu-nantes.fr

M. Coste-Burel

CHU de Nantes, Pôle Biologie, IFR 26, Service de Virologie,

EA 4271, 44093 Nantes Cedex 1, France

C. Laboisse

CHU de Nantes, Service d'Anatomie et Cytologie Pathologiques, 44093 Nantes Cedex 1, France

\section{A. Dahlab}

Sanofi Pasteur MSD, Lyon, France squamous cells of undetermined significance, $49 \%$ in lowgrade squamous intraepithelial lesion and $75 \%$ in highgrade squamous intraepithelial lesion (HSIL) $(p<0.001)$. Among 980 women with normal cytology, the overall HPV prevalence varied according to age from $44 \%$ below 20 years to about $10 \%$ above 35 years $(p<0.001)$. The most prevalent HPV genotype in all cytological diagnoses was HPV 16. HPV 53 appeared as the second most common genotype in normal cytological samples $(10.9 \%$ of HPV positive samples) but its prevalence decreased in HSIL to less than $4 \%$.

Conclusion The proportion of HR HPV positive women increased according to cytological diagnosis severity. HPV 16 appeared as the most commonly encountered genotype even when the diagnosis was normal. Its prevalence increased with diagnosis severity hereby confirming that HPV 16 is more aggressive than other genotypes.

Keywords Human papillomavirus - Genotype - Smears · Normal cytological diagnosis
Abbreviations
ASCUS Atypical squamous cells of undetermined significance
CIN Cervical intraepithelial neoplasia
HPV Human papillomavirus
HSIL High-grade squamous intraepithelial lesion
ICC Invasive squamous cell carcinoma
LSIL Low-grade squamous intraepithelial lesion

\section{Introduction}

Human papillomavirus (HPV) genital infection is highly prevalent among young sexually active women and represents 
the most common sexually transmitted infection [1-3]. It is today universally accepted that HPV is associated with the occurrence of cervical lesions, namely cervical intraepithelial neoplasia (CIN) and invasive squamous cell carcinoma (ICC) [4-6]. After infection, spontaneous viral clearance is observed in most cases within 2 years [7-9]. However, after persistent infection, cervical abnormalities may appear which may progress to low (CIN1) or high-grade CIN (CIN2/3) and ICC [10].

ICC represents the second most common cancer among women worldwide [11] and accounts for 3,400 new cases and about 1,000 deaths annually in France [12, 13]. Two VLP L1 HPV vaccines are now available: an HPV 16/18 bivalent vaccine, Cervarix ${ }^{\circledR}$ for the prevention of HPV 16/18-related cervical precancerous lesions and an HPV $6 / 11 / 16 / 18$ quadrivalent vaccine, Gardasil ${ }^{\circledR}$ which also protects against vulvar and vaginal precancerous lesions, and against HPV 6- and HPV 11-associated genital diseases [14]. Recent French studies (EDiTH) reported that HPV $6 / 11 / 16 / 18$ were found in $88 \%$ of cases with external acuminata condylomata [15], 34\% of low grade squamous intraepithelial lesions (LSIL) [16], 64\% of CIN2/3 [17] and $82 \%$ of ICC [18].

Pap smears are routinely used as secondary prevention of CIN2/3 and cervical cancer. Although HPV genotype distribution in specific cervical lesions has been widely studied, HPV overall prevalence and type-specific prevalence in samples collected during routine clinical practice remain poorly documented. The aim of this study was thus to describe the HPV genotype distribution with special focus on vaccinal HPV types in samples collected during routine gynaecologic follow-up at the University Hospital of Nantes, France.

\section{Materials and methods}

Data collection

Data from women seen at the Gynaecology outpatient department, Nantes University Hospital, France, between 2000 and 2006 for a routine clinical follow-up were retrospectively collected from the hospital database. Information had to include data on HPV genotype as well as a cytological or histological diagnosis.

\section{Cytological samples}

Pap samples were routinely collected at the Department of Gynaecology of the Nantes University Hospital as follows: women with no cervical screening within the past 3 years underwent a cervical smear performed using an Ayre spatula and a cytobrush whereas no smear was performed in women with screening history within the past 3 years, except in case of clinical signs such as inflammatory process or metrorrhagia.

Histological samples

Cervical biopsies were performed through colposcopy with a micro-clip $(2 \times 3 \mathrm{~mm})$. Colposcopy was indicated in case of abnormal smear such as atypical squamous cells of undetermined significance (ASCUS), LSIL, or high-grade squamous intraepithelial lesion (HSIL), or in presence of oncogenic HPV type. Biopsy samples were taken from the most acidophil area after applying acetic acid 3\%, or on the junctional area. Samples were immediately dropped in formaldehyde.

\section{Samples for HPV testing}

HPV testing was systematically performed as part of the routine gynaecologic follow-up, either during a screening visit or a follow-up for cervico-vaginal lesion. Cervical samples were collected with a bactopick swab or a cytobrush in $1 \mathrm{ml}$ of sterile phosphate buffered saline solution and transferred to the laboratory for HPV analysis.

HPV detection and genotyping

Total DNA was extracted from $200 \mu \mathrm{l}$ of the cell suspension using the MagNA Pure LC DNA isolation kit I (Roche diagnostics) and amplified by real-time SYBR green PCR with MY09/MY11 consensus primers on a rotorgen 3000 (Corbett Research). All samples were tested for the presence of human albumin gene by real-time PCR as described previously to assess the sample quality [19].

Genotyping was performed by sequencing after purification of the PCR amplified product using the ABI PRISM 3100 (Applied Biosystems) with Big Dye terminator kit (Applera). Sequences were then submitted to the NCBI BLAST. HPV 16, 18, 31, 33, 35, 39, 45, 52, 53, 58, 66, 68, 73 and 82 were classified as high-risk genotypes whereas HPV 6, 11, 44, 54, 61, 62, 70, 71, 72, 81, 83, 84 and 87 were considered as low-risk genotypes.

Selection of swabs and biopsies

Since mean time to HPV clearance after infection ranges from about 13 to 19 months [9], we only considered cervical swabs or biopsies collected no more than 12 months before HPV genotyping. Therefore, for each woman, samples collected more than 12 months before genotyping and samples collected after HPV genotyping (with a 20 days margin) were not considered for the analysis. Women who underwent treatment for their cervical lesion prior to HPV 


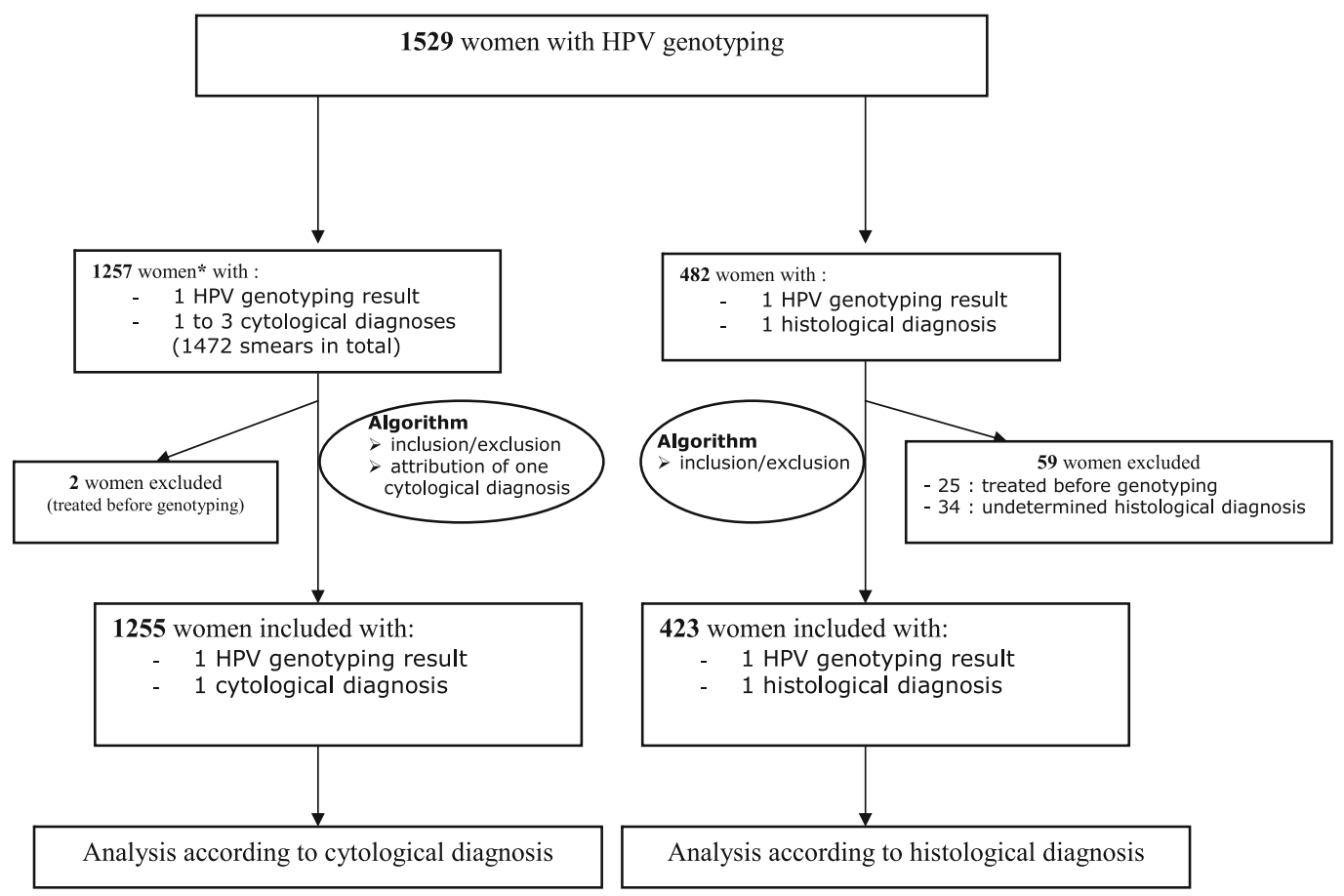

* 210 women had simultaneously one cytological and one histological diagnosis

Fig. 1 Inclusion algorithm

genotyping were also excluded from the analysis. The inclusion algorithm is presented on Fig. 1.

Cytological and histological diagnoses

Cytological specimens were classified according to the 2001 Bethesda classification [20] as follows: normal, ASCUS, LSIL, or HSIL. When several cytological samples were available for a single woman, the most pejorative diagnosis was kept for the analysis. Histological specimens were classified according to the Richart classification [21] as either normal, CIN1, CIN2, CIN3, or ICC.

Two separate analyses of HPV genotype distribution were conducted, one on women with available cytological diagnosis, the other on women with available histological data.

\section{Statistical analysis}

Qualitative variables were described with the number of cases and the proportions. For quantitative variables, mean and range were given. A $\chi^{2}$ for trend analysis was performed to study the association between HPV positivity and severity of diagnosis. Statistical analysis was performed with $\mathrm{SAS}^{\circledR}$ v.8.02 and $\mathrm{AdClin}^{\circledR}$ v.3.1. The ratio of HPV 16 prevalence in CIN3 versus CIN1 and versus normal histology together with its $95 \%$ confidence interval was calculated to appreciate the importance of this genotype in the risk of severe histological lesions.

Ethics and consent

According to the French legislation (Public Health Code modified by the law No. 2004-806, 9 August 2004 and the Huriet-Sérusclat act 88-1138, 20 December 1988, and since this study was conducted during patients' routine clinical follow-up without any modification, no ethics committee approval was necessary. All women gave their oral consent for HPV testing.

\section{Results}

A total of 1,257 women were included in the analysis according to cytological diagnosis. In parallel, 482 women were included according to histological diagnosis.

Results according to cytological diagnosis

Among women with cytological diagnosis, two were excluded because they underwent cervical treatment prior to HPV genotyping. Mean age of the 1,255 remaining women was 37.5 years (range 15-86) and varied significantly from 32 years in LSIL to 38.5 years in HSIL 
( $p<0.001)$. The mean delay between cytological diagnosis and genotyping was $12 \pm 40$ days. In 980 women (78\%), the cytological diagnosis was normal whereas $141(11 \%)$ had an ASCUS, 98 (8\%) an LSIL, and 36 (3\%) an HSIL (Table 1).

\section{Overall HPV prevalence}

Among all 1,255 women, 265 (21\%) were HPV positive. This proportion increased significantly according to cytological diagnosis severity from $13 \%$ in normal specimens to $30 \%$ in ASCUS, $69 \%$ in LSIL and $75 \%$ in HSIL ( $\chi^{2}$ for trend analysis gave $p<0.001$; Table 2). Multiple HPV infection was present in approximately $6 \%$ of all HPV positive samples with a higher prevalence in ASCUS (17\%).
Among the $265 \mathrm{HPV}$ positive samples, $184(69 \%)$ had at least one high-risk genotype.

Women with normal cytological diagnosis

Mean age of women with normal cytological diagnosis was 38.5 years. About $13 \%$ of these 980 women were HPV positive, $3 \%$ of them as a multiple HPV infection. The proportion of HPV positive women varied significantly according to age with the highest prevalence $(44 \%)$ observed below 20 years of age. Thereafter, the prevalence decreased with increasing age reaching about $10 \%$ above 35 years $(p<0.001)$. Eighty women $(8.2 \%)$ had at least one highrisk genotype. This proportion decreased significantly with age from $24 \%$ in women below 25 years of age to $6.5 \%$ in

Table 1 Characteristics of women with normal cytological diagnosis, atypical squamous cells of undetermined significance (ASCUS), low-grade squamous intraepithelial lesion (LSIL), or high-grade squamous intraepithelial lesion (HSIL)

\begin{tabular}{|c|c|c|c|c|c|}
\hline & \multicolumn{4}{|c|}{ Cytological diagnosis } & \multirow[t]{2}{*}{ Total } \\
\hline & Normal & ASCUS & LSIL & HSIL & \\
\hline Number of women (\%) & $980(78.1)$ & $141(11.2)$ & $98(7.8)$ & $36(2.9)$ & $1,255(100)$ \\
\hline Mean age in years (range) & $38.5(17-86)$ & $33.9(16-80)$ & $32.0(15-58)$ & $38.5(19-73)$ & $37.5(15-86)$ \\
\hline \multicolumn{6}{|l|}{ Age distribution, $n(\%)$} \\
\hline$<20$ years & $18(1.8)$ & $6(4.3)$ & $6(6.1)$ & $1(2.8)$ & $31(2.5)$ \\
\hline $20-24$ & $61(6.2)$ & $26(18.4)$ & $21(21.4)$ & $2(5.6)$ & $110(8.8)$ \\
\hline $25-29$ & $121(12.3)$ & $21(14.9)$ & $20(20.4)$ & $5(13.9)$ & $167(13.3)$ \\
\hline $30-34$ & $199(20.3)$ & $25(17.7)$ & $13(13.3)$ & $8(22.2)$ & $245(19.5)$ \\
\hline $35-39$ & $165(16.8)$ & $23(16.3)$ & $17(17.3)$ & $4(11.1)$ & 209 (16.7) \\
\hline $40-44$ & $158(16.1)$ & $20(14.2)$ & $11(11.2)$ & $6(16.7)$ & $195(15.5)$ \\
\hline $45-49$ & $95(9.7)$ & $8(5.7)$ & $5(5.1)$ & $4(11.1)$ & $112(8.9)$ \\
\hline$\geq 50$ & $163(16.6)$ & $12(8.5)$ & $5(5.1)$ & $6(16.7)$ & $186(14.8)$ \\
\hline
\end{tabular}

Percentages are given within parentheses

Table 2 Overall HPV prevalence and prevalence of HPV 6, 11, 16, and 18 among women with normal cytological diagnosis, ASCUS, LSIL, or HSIL

\begin{tabular}{|c|c|c|c|c|c|}
\hline & \multirow{2}{*}{$\begin{array}{l}\text { Total samples } \\
(n=1,255)\end{array}$} & \multicolumn{4}{|c|}{ Cytological diagnosis } \\
\hline & & Normal $(n=980)$ & ASCUS $(n=141)$ & LSIL $(n=98)$ & HSIL $(n=36)$ \\
\hline HPV positive & $265(21.1)(100)$ & $128(13.1)(100)$ & $42(29.8)(100)$ & $68(69.4)(100)$ & $27(75.0)(100)$ \\
\hline High-risk HPV ${ }^{a}$ & $184(14.7)(69.4)$ & $80(8.2)(62.5)$ & $29(20.6)(69.0)$ & $48(49.0)(70.6)$ & $27(75.0)(100)$ \\
\hline Low-risk HPV ${ }^{b}$ & $65(5.2)(24.5)$ & $34(3.5)(26.6)$ & $12(8.5)(28.6)$ & $19(19.4)(27.9)$ & $0(0.0)(0.0)$ \\
\hline HPV 6 total & $26(2.1)(9.8)$ & $10(1.0)(7.8)$ & $7(5.0)(16.7)$ & $9(9.2)(13.2)$ & $0(0.0)(0.0)$ \\
\hline HPV 11 total & $6(0.5)(2.3)$ & $2(0.2)(1.6)$ & $2(1.4)(4.8)$ & $2(2.0)(2.9)$ & $0(0.0)(0.0)$ \\
\hline HPV 16 total & $90(7.2)(34.0)$ & $35(3.6)(27.3)$ & $19(13.5)(45.2)$ & $20(20.4)(29.4)$ & $16(44.4)(59.3)$ \\
\hline HPV 18 total & $12(1.0)(4.5)$ & $6(0.6)(4.7)$ & $0(0.0)(0.0)$ & $4(4.1)(5.9)$ & $2(5.6)(7.4)$ \\
\hline HPV 16 and/or 18 total & $100(8.0)(37.7)$ & $40(4.1)(31.3)$ & $19(13.5)(45.2)$ & $23(23.5)(33.8)$ & $18(50.0)(66.7)$ \\
\hline
\end{tabular}

The number of cases is given as well as the prevalence among all samples (within parentheses) and the prevalence among HPV positive women (within parentheses and italics)

${ }^{\text {a }}$ Women infected by at least one high-risk genotype

${ }^{\mathrm{b}}$ Women infected by one or several low-risk genotype(s) without high-risk genotype 
Fig. 2 Distribution of high-risk and low-risk HPV genotypes according to age in women with normal cytological diagnosis $(n=980)$

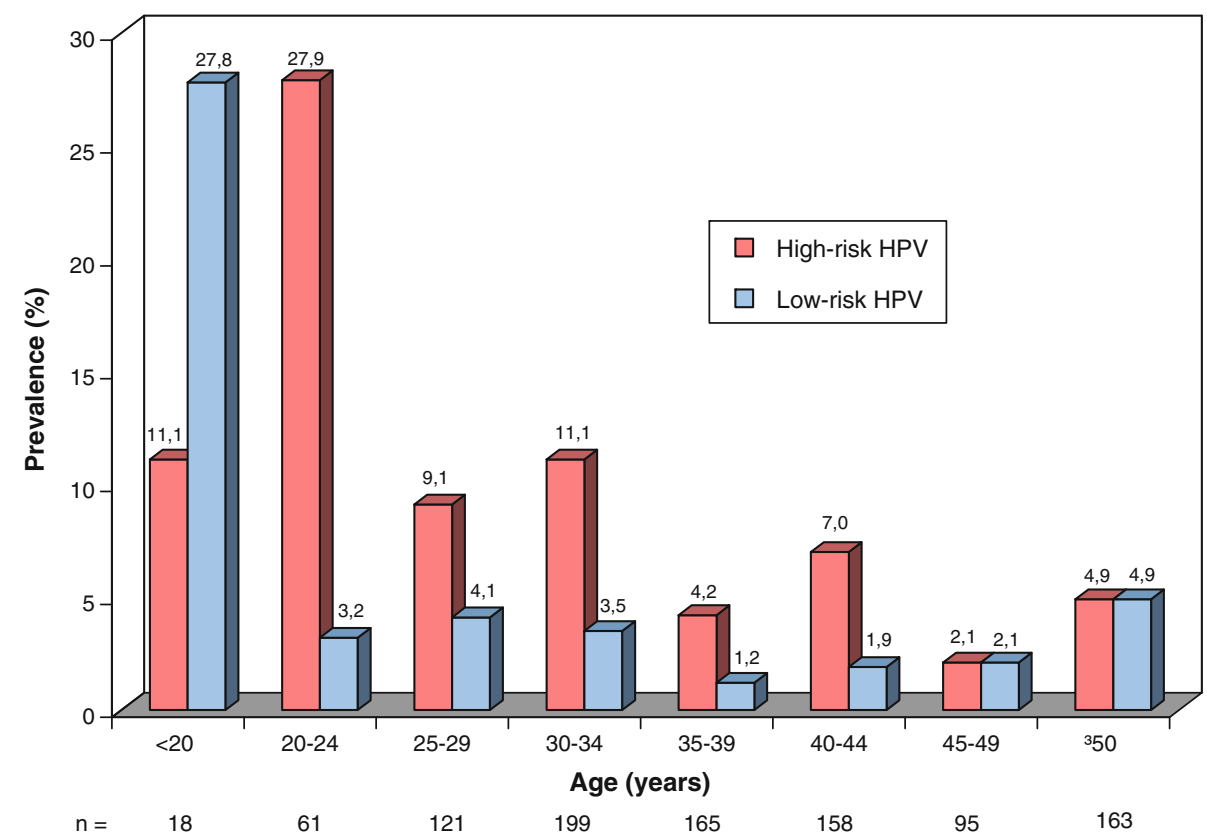

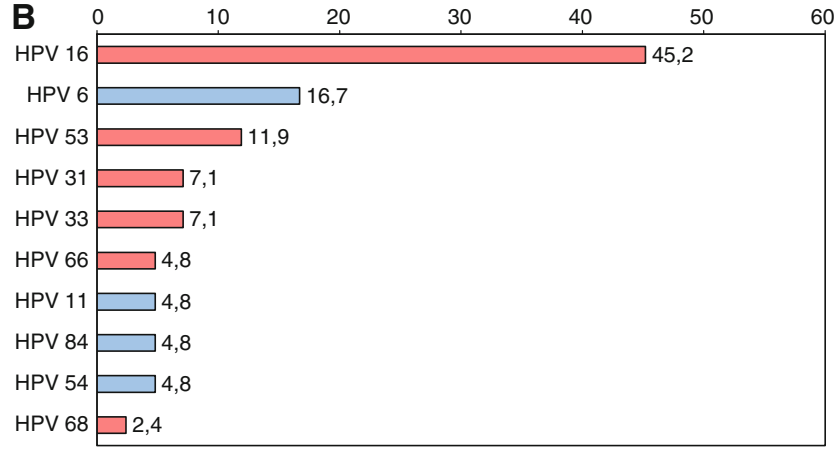

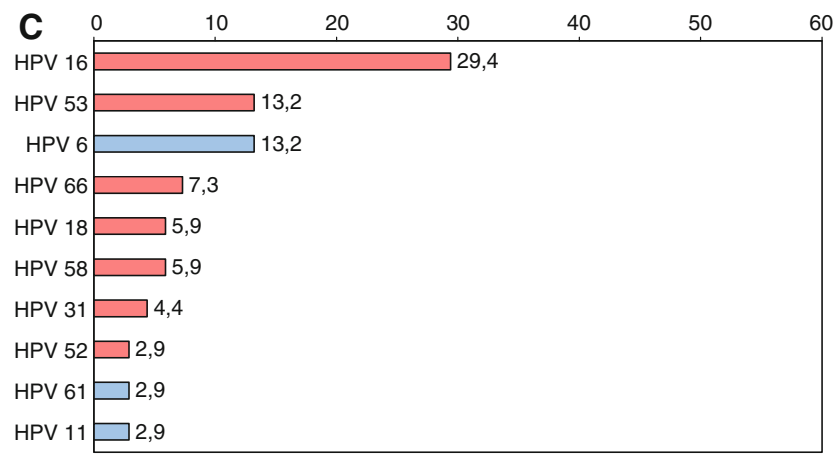

Fig. 3 Prevalence of most frequently encountered HPV genotypes in cervical samples with normal cytological diagnosis (a), ASCUS (b), LSIL (c) and HSIL (d). Red bars indicate high-risk genotypes, blue

women over 25 years $(p<0.001$; Fig. 2$)$. The most prevalent HPV genotypes were by descending order of frequency HPV 16 (3.6\%), HPV 53 (1.4\%), HPV 6 (1.0\%), HPV 31 $(0.9 \%)$ and HPV $33(0.7 \%)$ (Fig. 3). The prevalence of HPV 6, 11, 16, and 18 is given in Table 2. At least one of these four genotypes, associated or not with another HPV type was present in $5.3 \%$ of samples with normal cytology.

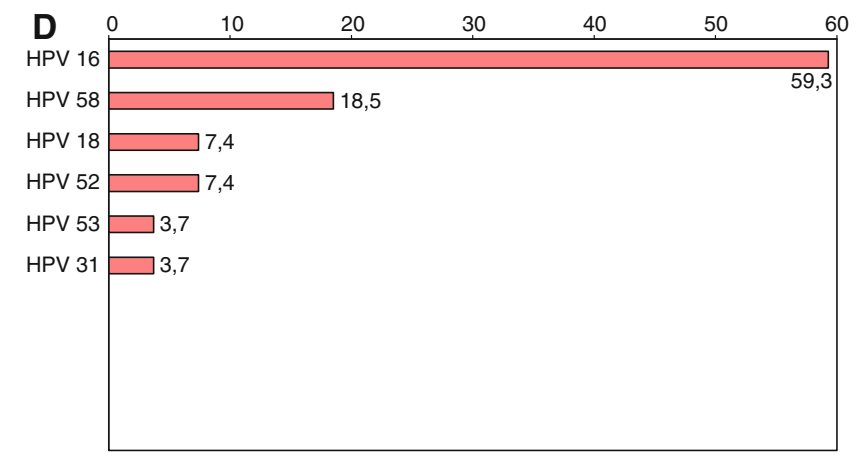

bars low-risk genotypes. Prevalence is given as the proportion of each specific HPV genotype among HPV positive samples

\section{Women with ASCUS diagnosis}

Mean age of the 141 women with an ASCUS was 34 years. Among these women, 42 (30\%) were HPV positive, of whom $35(83 \%)$ were infected by a single HPV type. Among HPV positive women with ASCUS, 69\% had at least one high-risk genotype. The most prevalent HPV 
types were HPV $16(n=19,13 \%$ of all ASCUS, $45 \%$ of HPV positive ASCUS), HPV $6(n=7)$, HPV $53(n=5)$, HPV 31 and HPV 33 ( $n=3$ ) (Fig. 3). HPV 6 and/or 11 and/or 16 and/or 18 were present in $20 \%$ of all ASCUS cases and $67 \%$ of HPV positive ASCUS.

\section{Women with LSIL diagnosis}

Women with LSIL diagnosis were younger than the average with a mean age of 32 years. Among these 98 women, $69 \%$ were HPV positive of whom $93 \%$ as a monoinfection. Seventy-one percent of HPV positive LSIL cases were infected with at least one high-risk HPV type. Most frequently encountered HPV genotypes in LSIL were HPV 16 ( $n=20,20 \%$ of all LSIL, 29\% of HPV positive LSIL), HPV 53 and HPV $6(n=9)$, HPV $66(n=5)$ and HPV 18 $(n=4)$. Alone or in association with other HPV types, HPV 6 and/or 11 and/or 16 and/or 18 were found in 35\% of all LSIL and $50 \%$ of HPV positive LSIL cases.

\section{Women with HSIL diagnosis}

Thirty-six women had an HSIL diagnosis. Mean age was 38.5 years. Seventy-five percent were HPV positive, almost all of them (96\%) with an HPV monoinfection. All HPV positive HSIL specimens were infected by at least one high-risk HPV genotype. Most frequent genotypes were HPV 16 ( $n=16,44 \%$ of all HSIL, 59\% of HPV positive HSIL), HPV $58(n=5), \mathrm{HPV} 18$ and HPV $52(n=2)$ (Fig. 3). HPV 6 and/or 11 and/or 16 and/or 18 were found in $50 \%$ of all HSIL and in $67 \%$ of HPV positive HSIL.

Results according to histological diagnosis

Among women with histological data, 59 were excluded, 25 because of cervical treatment prior to HPV genotyping and 34 because of undetermined histological diagnosis (Fig. 1). Characteristics of the 423 remaining women are presented in Table 3. Mean age was 34 years (range 16-64) and varied from 32.5 years in CIN1 to 44 years in women with cancer diagnosis. The mean delay between biopsy and HPV genotyping was 15.5 days (range -20 to 363). In 163 women (39\%), the diagnosis was normal whereas 117 women (28\%) had a CIN1, 79 (19\%) had a CIN2 and $62(15 \%)$ had a CIN3. Only two cases had an ICC diagnosis.

\section{Overall HPV prevalence}

Among all 423 women, 228 (54\%) were HPV positive. This proportion increased significantly with increasing diagnosis severity from $33 \%$ in normal histological specimens, $55 \%$ in CIN1, 68\% in CIN2, and $89 \%$ in CIN3 ( $\chi^{2}$ for trend gave $p<0.001$; Table 4). Multiple HPV infection was found in approximately $11 \%$ of HPV positive cases with the highest prevalence observed in CIN2 (17\%). Among HPV positive specimens, $85 \%$ were infected by at least one high-risk type.

Women with normal histological diagnosis

Histological diagnosis was normal in 163 women (mean age 34.7 years) of whom $54(33 \%)$ were HPV positive. Among these HPV positive women, 91\% were infected by a single HPV type. At least one high-risk genotype was found in $24 \%$ of biopsies with normal diagnosis. The most common HPV genotypes were HPV $16(n=10,6 \%$ of normal biopsies, $19 \%$ of HPV positive normal biopsies), HPV $53(n=9)$, HPV 18 and HPV $6 \quad(n=6)$ (Fig. 4). HPV 6, 11, 16 and/or 18 were found in $14 \%$ of normal histological specimens and $43 \%$ of HPV positive normal biopsies.

Table 3 Characteristics of women with normal histological diagnosis, low-grade cervical intraepithelial neoplasia (CIN1), CIN2, CIN3, or invasive squamous cell carcinoma (ICC)

\begin{tabular}{|c|c|c|c|c|c|c|}
\hline & \multicolumn{5}{|c|}{ Histological diagnosis } & \multirow[t]{2}{*}{ Total } \\
\hline & Normal & CIN1 & CIN2 & CIN3 & ICC & \\
\hline Number of women (\%) & $163(38.5)$ & $117(27.7)$ & 79 (18.7) & $62(14.7)$ & $2(0.5)$ & $423(100)$ \\
\hline Mean age in years (range) & $34.7(16-64)$ & $32.6(17-60)$ & $32.8(18-54)$ & $36.1(19-57)$ & $44.0(31-57)$ & $34.0(16-64)$ \\
\hline \multicolumn{7}{|l|}{ Age distribution, $n(\%)$} \\
\hline$<20$ years & $4(2.5)$ & $7(6.0)$ & $2(2.5)$ & $1(1.6)$ & $0(0.0)$ & $14(3.3)$ \\
\hline $20-24$ & $24(14.7)$ & $22(18.8)$ & $12(15.2)$ & $5(8.1)$ & $0(0.0)$ & $63(14.9)$ \\
\hline $25-29$ & $30(18.4)$ & $18(15.4)$ & $17(21.5)$ & $9(14.5)$ & $0(0.0)$ & $74(17.5)$ \\
\hline $30-34$ & $34(20.9)$ & $23(19.7)$ & $14(17.7)$ & $12(19.4)$ & $1(50.0)$ & $84(19.9)$ \\
\hline $35-39$ & $20(12.3)$ & $20(17.1)$ & $17(21.5)$ & $14(22.6)$ & $0(0.0)$ & $71(16.8)$ \\
\hline$\geq 40$ & $51(31.3)$ & $27(23.1)$ & $17(21.5)$ & $21(33.9)$ & $1(50.0)$ & $117(27.7)$ \\
\hline
\end{tabular}

Percentages are given within parentheses 
Table 4 Overall HPV prevalence and prevalence of HPV 6, 11, 16, and 18 among women with normal histological diagnosis, CIN1, CIN2, or CIN3

\begin{tabular}{|c|c|c|c|c|c|}
\hline & \multirow{2}{*}{$\begin{array}{l}\text { Total samples } \\
(n=423)\end{array}$} & \multicolumn{4}{|c|}{ Histological diagnosis } \\
\hline & & Normal $(n=163)$ & CIN1 $(n=117)$ & CIN2 $(n=79)$ & CIN3 $(n=62)$ \\
\hline HPV positive & $228(53.9)(100)$ & $54(33.1)(100)$ & $64(54.7)(100)$ & $54(68.4)(100)$ & $55(88.7)(100)$ \\
\hline High-risk $\mathrm{HPV}^{\mathrm{a}}$ & $193(45.6)(84.6)$ & $39(23.9)(72.2)$ & $47(40.2)(73.4)$ & $54(68.4)(100)$ & $53(85.5)(96.4)$ \\
\hline Low-risk HPV ${ }^{b}$ & $29(6.9)(12.7)$ & $13(8.0)(24.1)$ & $14(12.0)(21.9)$ & $0(0.0)(0.0)$ & $2(3.2)(3.6)$ \\
\hline HPV 6 total & $15(3.5)(6.6)$ & $6(3.7)(11.1)$ & $7(6.0)(10.9)$ & $2(2.5)(3.7)$ & $0(0.0)(0.0)$ \\
\hline HPV 11 total & $3(0.7)(1.3)$ & $1(0.6)(1.9)$ & $2(1.7)(3.1)$ & $0(0.0)(0.0)$ & $0(0.0)(0.0)$ \\
\hline HPV 16 total & $88(20.8)(38.6)$ & $10(6.1)(18.5)$ & $17(14.5)(26.6)$ & $25(31.6)(46.3)$ & $35(56.5)(63.6)$ \\
\hline HPV 18 total & $19(4.5)(8.3)$ & $6(3.7)(11.1)$ & $5(4.3)(7.8)$ & $7(8.9)(13.0)$ & $1(1.6)(1.8)$ \\
\hline HPV 16 and/or 18 total & $105(24.8)(46.1)$ & $23(14.1)(42.6)$ & $21(17.9)(32.8)$ & $31(39.2)(57.4)$ & $36(58.1)(65.5)$ \\
\hline
\end{tabular}

The number of cases is given as well as the prevalence among all samples (within parentheses) and the prevalence among HPV positive women (within parentheses and italics)

${ }^{\text {a }}$ Women infected by at least one high-risk genotype

${ }^{\mathrm{b}}$ Women infected by one or several low-risk genotype(s) without high-risk genotype

\begin{tabular}{|c|c|c|c|c|c|c|}
\hline A & 10 & 20 & 30 & 40 & 50 & 60 \\
\hline HPV 16 & & 18,5 & & & & \\
\hline HPV 53 & 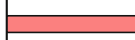 & 6,7 & & & & \\
\hline HPV 18 & 1 & & & & & \\
\hline HPV 6 & $\longrightarrow$ & & & & & \\
\hline HPV 62 & 7,4 & & & & & \\
\hline HPV 31 & 5,6 & & & & & \\
\hline HPV 58 & $\Longrightarrow$ 5,6 & & & & & \\
\hline HPV 61 & $5^{5,6}$ & & & & & \\
\hline HPV 66 & $\square_{3,7}$ & & & & & \\
\hline HPV 33 & $\square^{3,7}$ & & & & & \\
\hline
\end{tabular}

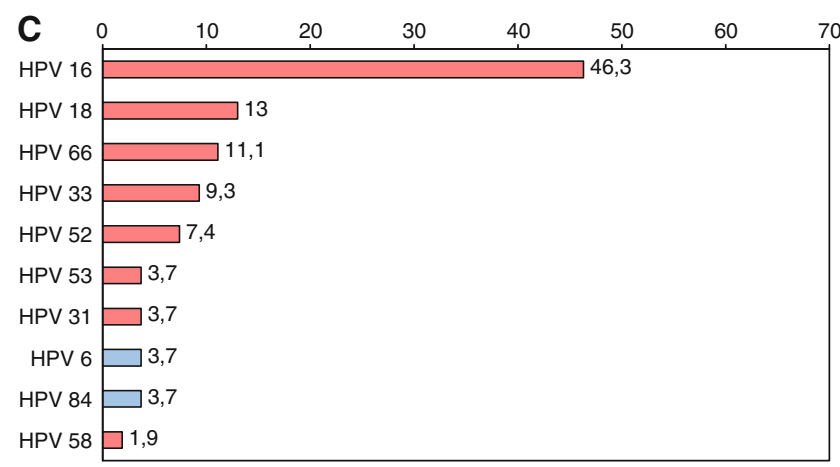

Fig. 4 Prevalence of most frequently encountered HPV genotypes in cervical samples with normal histological diagnosis (a), CIN1 (b), CIN2 (c) and CIN3 (d). Red bars indicate high-risk genotypes, blue

\section{Women with CIN1 diagnosis}

A CIN1 diagnosis was found in 117 women (mean age 32.6 years) of whom $64(55 \%)$ were HPV positive. Among these HPV positive women, $90 \%$ had a monoinfection. At least one high-risk genotype was found in $40 \%$ of CIN1. Most frequent genotypes were HPV $16(n=17,15 \%$ of CIN1, 27\% of HPV positive CIN1), HPV $53(n=10), \mathrm{HPV}$

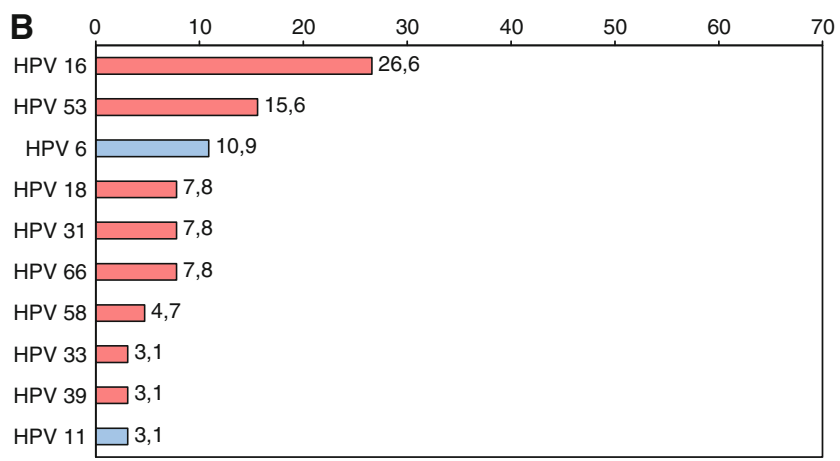

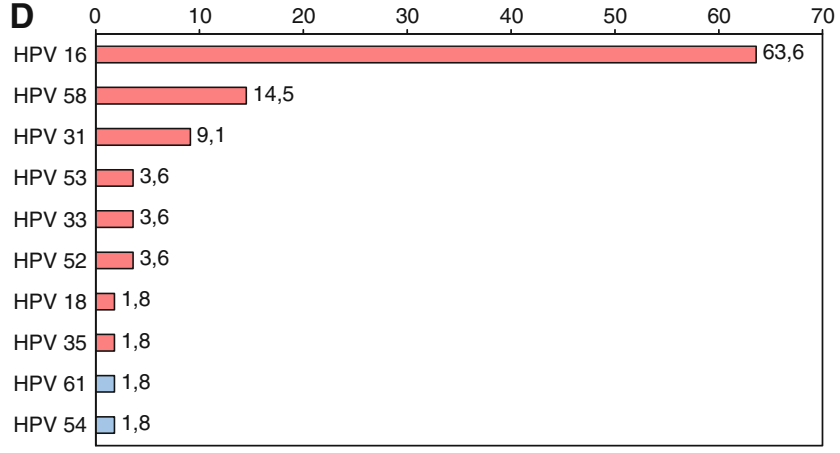

bars low-risk genotypes. Prevalence is given as the proportion of each specific HPV genotype among HPV positive samples

$6(n=7)$, and HPV 18, 31, and $66(n=5)$. HPV 6, 11, 16 and/or 18 were present in $26 \%$ of CIN1 and $47 \%$ of HPV positive CIN1 specimens.

Women with CIN2 or CIN3 diagnosis

CIN2 and CIN3 were found in 79 and 62 women with histological diagnosis. Mean age was 32.8 and 36.1 years, 
respectively. Prevalence of HPV positive CIN2 and CIN3 specimens was 68 and $89 \%$, respectively (Table 4). Monoinfection was observed in $83 \%$ of HPV positive CIN2 and in $91 \%$ of CIN3. At least one high-risk genotype was found in $68 \%$ of CIN 2 and in $86 \%$ of CIN3 specimens. Most common HPV genotypes in CIN2 were HPV $16(n=25,32 \%$ of CIN2, $46 \%$ of HPV positive CIN2) followed by HPV 18 $(n=7)$, HPV $66(n=6)$ and HPV $33(n=5)$ (Fig. 4). In CIN3, HPV 16 was by far the most commonly observed genotype $(n=35,57 \%$ of CIN3, 64\% of HPV positive CIN3), followed by HPV $58(n=8)$, HPV $31(n=5)$ and HPV 53, HPV 33 and HPV $52(n=2)$. HPV 6 and/or 11 and/or 16 and/or 18 were found in $41 \%$ of CIN2 and 58\% of CIN 3 and in 59 and $65 \%$ of HPV positive CIN2 and CIN3, respectively. Only one case (CIN2) had a co-infection involving HPV 6 (or HPV 11) with a high-risk HPV type (HPV $6+$ HPV 39). The ratio of HPV 16 prevalence in CIN3 versus CIN1 was 3.89 (95\% CI [2.38-6.35]) and the ratio of HPV 16 prevalence in CIN3 versus normal histology 9.20 (95\% CI [4.86-17.4]).

\section{Discussion}

Based on a large number of subjects, our study reports the HPV overall prevalence and HPV genotype distribution in cytological and histological specimens collected in routine clinical practice in a hospital-based gynaecologic department.

Among all 1,255 women, the observed proportion of those with abnormal smears (22\%) is much higher than that previously reported by Bergeron et al. in France (3-4\%) $[22,23]$. This discrepancy may result from differences in the recruitment of patients. In each Bergeron study, approximately 200,000 smears were collected from public or private structures whereas in the present study, the recruitment was hospital-based with as a result, inclusion of more severe cases. Since it is hospital-based, our study population is thus not representative of the general population. However, our results depict university hospital routine clinical practice. Noteworthy, the diagnosis distribution among abnormal cytological samples is rather similar to Bergeron's data with approximately $50 \%$ of ASCUS, $36 \%$ of LSIL, and $13 \%$ of HSIL.

As expected, the prevalence of HPV positive samples increases with increasing diagnosis severity. In cytological samples, the overall HPV prevalence varies indeed from $13 \%$ in normal samples to $70-75 \%$ in LSIL and HSIL. In ASCUS cases, the prevalence is in-between at approximately $30 \%$. In histological samples, the overall HPV prevalence increases with the severity of the lesion reaching a maximum of $89 \%$ in CIN3 cases. The HPV prevalence observed in normal histological specimens (33\%) is higher than that in normal cytological samples (13\%). This may be explained by the fact that biopsy was performed in case of abnormal smear, clinical sign, or presence of oncogenic HPV type.

The overall HPV prevalence in women with normal cytological diagnosis observed in our study is twice higher than that reported in the US by Castle et al. in about 800,000 smears collected from women above 30 years of age [24]. The authors observed indeed that $4 \%$ of women with normal smears were carcinogenic HPV positives whereas the prevalence of high-risk HPV genotypes in this subgroup reached $8.2 \%$ in our cohort. This higher prevalence could be partly explained by the hospital-based recruitment in our study and by the different age distribution between cohorts. In France, a previous study based on 2,145 smears without lesion [25] collected from women above 30 years who underwent their biennial or triennial routine screening in the university hospital of Reims, reported that high-risk HPV genotypes were found in $10.8 \%$ of cases which is close to our findings. Another French study on 3,640 women (above 20 years of age) with normal cytology enrolled either from the university hospital of Amiens or from private gynaecologists, reported a highrisk HPV prevalence of $13.5 \%$ [26]. In a meta-analysis based on 157,879 women with normal cervical cytology [27], the overall HPV prevalence was estimated to be $10.4 \%$ (8\% in Europe). The high-risk HPV prevalence observed in our subgroup of women with normal cytological diagnosis appears thus similar to that reported earlier. In the present study, presence of high-risk genotypes in women with normal smears is highly associated with age with a prevalence decreasing from $24 \%$ to $6.5 \%$ before and after 25 years of age, respectively. Based on a rather large number of women with normal cytological diagnosis ( $n=980)$, our study stresses the fact that a substantial proportion of these women is infected by a high-risk HPV, this proportion exceeding $20 \%$ in women below 25 years of age. Such a high prevalence of HPV in younger women with normal cytology was already reported by De Sanjosé et al. [27] in the above mentioned meta-analysis.

On the other hand, the overall HPV prevalence in women with abnormal cytological or histological diagnosis is somehow lower in our study than what was previously reported in the literature. In CIN2/3 specimens for example, the observed prevalence of $77 \%$ is much lower than the 98\% reported by Pretet et al. [17]. The same is true for the HPV prevalence in LSIL (69 versus 98\%) [16]. A possible explanation is that in the present study, cytological or histological diagnosis and genotyping were possibly made on different samples and at different time points. These two parameters were associated with each other by linkage of the hospital genotype and diagnosis databases. Even if HPV infection is known to last for about 13-19 months [9], 
one can not exclude that HPV clearance occurred between diagnosis and genotyping. However, this hypothesis is unlikely since the mean delay between cytological diagnosis and genotyping (15 days) or between histological diagnosis and genotyping (11 days) is rather short. The HPV negative status of some cervical cell suspension specimens with severe dysplasia could instead result from a low HPV viral load or from the fact that some genotypes are not matched by the MY09/MY11 primers. A reasonable explanation for these discrepancies in HPV prevalence is indeed the use of HPV detection methods of different sensitivity [18]. Such differences in sensitivity and characteristics of PCR systems for the detection of HPV within clinical samples have been previously described and there is good evidence that some genotypes are better matched to the consensus primer pools than others [28-31]. To cope with these differences in sensitivity, we also provided genotypespecific prevalence data among HPV positive women.

HPV 16 was by far the most commonly encountered genotype in cervical smears and histological specimens even when the diagnosis was normal. Figures 3 and 4 clearly show that the prevalence of HPV 16 increases with increasing diagnosis severity hereby confirming that HPV 16 tends to persist longer and is more aggressive than other genotypes [32]. Since the overall HPV prevalence in women with abnormal smears in our study is lower than previously reported, we considered HPV positive samples to calculate the prevalence of each specific genotype. Doing so, the prevalence of HPV 16 in LSIL, CIN2 and CIN3 becomes of the same magnitude as what was previously observed [16, 17]. HPV 16 was indeed found in $29 \%$ of our LSIL cases which is very close to results reported in the meta-analysis by Clifford et al. (27\%) [33]. To illustrate the high pathogenic role of HPV 16, we calculated the ratio of HPV prevalence in CIN3 versus normal histology and versus CIN1. These ratios indicate that HPV 16 is almost fourfold more prevalent in CIN3 than in CIN1 and about ninefold more prevalent in CIN3 than in normal histological samples hereby confirming that this genotype is associated with lesions presenting a high probability of progression to severe forms. Although the number of cases is limited, HPV 53 appears as the second most common genotype in normal cytological samples and in LSIL (11 and 13\%, respectively) and as the third most common in ASCUS (12\%). However, the prevalence decreases in HSIL to less than $4 \%$, suggesting that samples infected with HPV 53 are at low risk of progression towards severe forms although some few cases of cervical cancer following HPV 53 monoinfection have been described [18]. The same phenomenon is observed for biopsies in which HPV 53 is frequently encountered in normal and CIN1 specimens $(16 \%)$ and much less prevalent in CIN2 and CIN3 (below 4\%). Alone or in association, HPV 6, 11, 16 and/or 18 are found in over
$50 \%$ of HPV positive cytological and histological specimens hereby suggesting that a substantial proportion of these lesions could be avoided by a quadrivalent HPV vaccination.

Despite some methodological limitations, our study provides accurate French data on HPV genotype distribution in samples collected during routine hospital-based gynaecologic follow-up and brings further evidence of the potential impact of a quadrivalent vaccination.

Acknowledgments The authors gratefully thank Pierre Pradat (pradat@univ-lyon1.fr) who provided medical writing services on behalf of Sanofi Pasteur MSD. Special thanks to Javier Velasco Martinez for his valuable help. The epidemiological part of this study was funded by Sanofi Pasteur MSD.

Conflict of interest André Dahlab is an employee of Sanofi Pasteur MSD.

Open Access This article is distributed under the terms of the Creative Commons Attribution Noncommercial License which permits any noncommercial use, distribution, and reproduction in any medium, provided the original author(s) and source are credited.

\section{References}

1. Dalstein V, Briolat J, Birembaut P, Clavel C (2006) The epidemiology of genital human papillomavirus infections. Rev Prat 56:1877-1881

2. Moscicki AB (2005) Impact of HPV infection in adolescent populations. J Adolesc Health 37:S3-S9

3. Wiley D, Masongsong E (2006) Human papillomavirus: the burden of infection. Obstet Gynecol Surv 61:S3-S14

4. Bosch FX, Lorincz A, Munoz N, Meijer CJ, Shah KV (2002) The causal relation between human papillomavirus and cervical cancer. J Clin Pathol 55:244-265

5. Bosch FX, Manos MM, Munoz N, Sherman M, Jansen AM, Peto $\mathrm{J}$ et al (1995) Prevalence of human papillomavirus in cervical cancer: a worldwide perspective. International biological study on cervical cancer (IBSCC) Study Group. J Natl Cancer Inst 87:796802

6. Walboomers JM, Jacobs MV, Manos MM, Bosch FX, Kummer JA, Shah KV et al (1999) Human papillomavirus is a necessary cause of invasive cervical cancer worldwide. J Pathol 189:1219

7. Holowaty P, Miller AB, Rohan T, To T (1999) Natural history of dysplasia of the uterine cervix. J Natl Cancer Inst 91:252-258

8. Insinga RP, Dasbach EJ, Elbasha EH, Liaw KL, Barr E (2007) Incidence and duration of cervical human papillomavirus 6,11 , 16 , and 18 infections in young women: an evaluation from multiple analytic perspectives. Cancer Epidemiol Biomarkers Prev 16:709-715

9. Trottier H, Mahmud S, Prado JC, Sobrinho JS, Costa MC, Rohan TE et al (2008) Type-specific duration of human papillomavirus infection: implications for human papillomavirus screening and vaccination. J Infect Dis 197:1436-1447

10. Moscicki AB, Schiffman M, Kjaer S, Villa LL (2006) Chapter 5: updating the natural history of HPV and anogenital cancer. Vaccine 24(Suppl 3):S42-S51

11. Parkin DM, Bray F, Ferlay J, Pisani P (2005) Global cancer statistics, 2002. CA Cancer J Clin 55:74-108 
12. Belot A, Grosclaude P, Bossard N, Jougla E, Benhamou E, Delafosse P (2010) Cancer incidence and mortality in France over the period 1980-2005. Rev Épidemiol Santé Publique (in press)

13. Guizard AV, Trétarre B (2008) Col de l'utérus. In: Réseau français des registres de cancer, Hospices civils de Lyon, Inserm, Invs (eds) Estimation de l'incidence et de la mortalité par cancer en France de 1980 à 2005. Francim, HCL, Inserm, InVS. Saint-Maurice. http://www.invs.sante.fr/surveillance/cancers/estimations_cancers. Consulté le 21 May 2008

14. Villa LL, Costa RL, Petta CA, Andrade RP, Ault KA, Giuliano AR et al (2005) Prophylactic quadrivalent human papillomavirus (types 6, 11, 16, and 18) L1 virus-like particle vaccine in young women: a randomised double-blind placebo-controlled multicentre phase II efficacy trial. Lancet Oncol 6:271-278

15. Aubin F, Pretet JL, Jacquard AC, Saunier M, Carcopino X, Jaroud $F$ et al (2008) Human papillomavirus genotype distribution in external acuminata condylomata: a Large French National Study (EDiTH IV). Clin Infect Dis 47:610-615

16. Pretet JL, Jacquard AC, Saunier M, Clavel C, Dachez R, Gondry J et al (2008) Human papillomavirus genotype distribution in lowgrade squamous intraepithelial lesions in France and comparison with CIN2/3 and invasive cervical cancer: the EDiTH III study. Gynecol Oncol 110:179-184

17. Pretet JL, Jacquard AC, Carcopino X, Monnier-Benoit S, Averous G, Soubeyrand B et al (2008) Human papillomavirus genotype distribution in high grade cervical lesions (CIN 2/3) in France: EDITH study. Int J Cancer 122:424-427

18. Pretet JL, Jacquard AC, Carcopino X, Charlot JF, Bouhour D, Kantelip B et al (2008) Human papillomavirus (HPV) genotype distribution in invasive cervical cancers in France: EDITH study. Int J Cancer 122:428-432

19. Laurendeau I, Bahuau M, Vodovar N, Larramendy C, Olivi M, Bieche I et al (1999) TaqMan PCR-based gene dosage assay for predictive testing in individuals from a cancer family with INK4 locus haploinsufficiency. Clin Chem 45:982-986

20. Solomon D, Davey D, Kurman R, Moriarty A, O'Connor D, Prey M et al (2002) The 2001 Bethesda System: terminology for reporting results of cervical cytology. JAMA 287:2114-2119

21. Tumours of the uterine cervix. In: F Tavassoli, P Devilee (eds) Tumours of the breast and female genital organs. WHO classification of tumours. IARC Press, Lyon, 2003

22. Bergeron C, Cartier I, Guldner L, Lassalle M, Savignoni A, Asselain B (2005) Lésions précancéreuses et cancers du col de l'utérus diagnostiqués par le frottis cervical. Ile-de-France, enquête Crisap, 2002. Bull Epidemiol Hebd 2:5-6

23. Bergeron C, Cohet C, Bouée S, Lorans C, Rémy V (2007) Management of abnormal smears intraepithelial neoplasia and associated treatment costs in France. Bull Epidemiol Hebd 1:4-6

24. Castle PE, Fetterman B, Poitras N, Lorey T, Shaber R, Kinney W (2009) Five-year experience of human papillomavirus DNA and Papanicolaou test cotesting. Obstet Gynecol 113:595-600

25. Clavel C, Masure M, Bory JP, Putaud I, Mangeonjean C, Lorenzato $M$ et al (2001) Human papillomavirus testing in primary screening for the detection of high-grade cervical lesions: a study of 7932 women. Br J Cancer 84:1616-1623

26. Boulanger JC, Sevestre H, Bauville E, Ghighi C, Harlicot JP, Gondry J (2004) Epidemiology of HPV infection. Gynecol Obstet Fertil 32:218-223

27. de Sanjose S, Diaz M, Castellsague X, Clifford G, Bruni L, Munoz $\mathrm{N}$ et al (2007) Worldwide prevalence and genotype distribution of cervical human papillomavirus DNA in women with normal cytology: a meta-analysis. Lancet Infect Dis 7:453-459

28. Coutlee F, Gravitt P, Kornegay J, Hankins C, Richardson H, Lapointe $\mathrm{N}$ et al (2002) Use of PGMY primers in L1 consensus PCR improves detection of human papillomavirus DNA in genital samples. J Clin Microbiol 40:902-907

29. Gravitt PE, Coutlee F, Iftner T, Sellors JW, Quint WG, Wheeler CM (2008) New technologies in cervical cancer screening. Vaccine 26(Suppl 10):K42-K52

30. Gravitt PE, Peyton CL, Alessi TQ, Wheeler CM, Coutlee F, Hildesheim A et al (2000) Improved amplification of genital human papillomaviruses. J Clin Microbiol 38:357-361

31. Qu W, Jiang G, Cruz Y, Chang CJ, Ho GY, Klein RS et al (1997) PCR detection of human papillomavirus: comparison between MY09/MY11 and GP5+/GP6+ primer systems. J Clin Microbiol 35:1304-1310

32. Munoz N, Hernandez-Suarez G, Mendez F, Molano M, Posso H, Moreno V et al (2009) Persistence of HPV infection and risk of high-grade cervical intraepithelial neoplasia in a cohort of Colombian women. Br J Cancer 100:1184-1190

33. Clifford GM, Rana RK, Franceschi S, Smith JS, Gough G, Pimenta JM (2005) Human papillomavirus genotype distribution in lowgrade cervical lesions: comparison by geographic region and with cervical cancer. Cancer Epidemiol Biomarkers Prev 14:1157-1164 